\title{
Properties of semi primitive roots
}

\author{
Dr.K.Vijaya lakshmi \\ Head, Department of Mathematics, Loyola Academy, Secunderabad.
}

\begin{abstract}
We know that the smallest positive integer $f$ such that $a^{f} \equiv 1 \bmod m$ is called the exponent of ' $a$ ' modulo $m$ and is denoted by $\exp _{m} a$. We say that ' $a$ ' is a semi-primitive root $\bmod m$ if $\exp _{m} a=\frac{\phi(m)}{2}$. In this paper we discuss the properties of the semi primitive roots and examine for which prime 2 is a semi-primitive root. If $S$ is the sum of semi-primitive roots less than $p$ then we proved that $S \equiv \mu\left(\frac{p-1}{2}\right) \bmod p$.Also we proved that if ' $a$ ' is a semi primitive root then ' $a$ ' is a quadratic residue, converse need not be true. It was established that whenever $a$ is a semi-primitive root mod $p$ where $p$ is of the form $4 n+3$ then $-a$ is a semi primitive root and if $p=4 n+1$ then $\exp _{m}(-a)=\frac{p-1}{4}$. We establish that 2 is semi-primitive root for mod $p$ whenever ' $p$ ' is of the form $2 q+1$ where ' $q$ ' is an odd prime of the form $4 n+3$ and if $4 n+1,8 n+3$ are primes

then -2 is a semi-primitive root mod $8 n+3$ by using Gauss Lemma [1].
\end{abstract}

Definition: Suppose ' $a$ ' is any integer and $\mathrm{m}$ is a positive integer such that $(\mathrm{a}, \mathrm{m})=1$.

We say that $\mathrm{a}$ is a semi-primitive root $\bmod \mathrm{m}$ if $\exp _{\mathrm{m}} \mathrm{a}=\phi(\mathrm{m}) / 2$. From the definition

It is clear that $a, a^{2}, \ldots . a^{\frac{\phi(m)}{2}}$ are in congruent mod $m$ and they form a cyclic sub group of reduced residue system mod $\mathrm{m}$.

Theorem 1: If $m$ has a primitive root then there are exactly $\frac{\phi(\phi(m))}{2}$ semi primitive roots given by $\mathrm{S}=\left\{a^{n}: 1 \leq n<\frac{\phi(m)}{2},\left(n, \frac{\phi(m)}{2}\right)=1\right\}$

Proof: If a is a semi-primitive root then

$\exp _{\mathrm{m}} a=\exp _{\mathrm{m}} a^{n}=\frac{\phi(m)}{2} \Leftrightarrow\left(n, \frac{\phi(m)}{2}\right)=1$

So every member of $\mathrm{S}$ is a semi-primitive root $\bmod \mathrm{m}$. Conversely, if ' $\mathrm{g}$ ' is a semi-primitive root then

$$
\begin{aligned}
& \exp _{\mathrm{m}} a^{k}=\exp _{\mathrm{m}} g=\frac{\phi(m)}{2} \text { for } 1 \leq k \leq \frac{\phi(m)}{2} \\
& \therefore\left(k, \frac{\phi(m)}{2}\right)=1
\end{aligned}
$$

Now we find the sum of semi-primitive roots less than ' $p$ '.

Theorem $2:$ If $\mathrm{p}$ is an odd prime and $\mathrm{S}$ is the sum of semi-primitive roots less than $\mathrm{p}$ then

$$
\mathrm{S} \equiv \mu\left(\frac{\mathrm{p}-1}{2}\right) \bmod p
$$

Proof: Suppose ' $a$ ' is a semi-primitive root $\bmod p$, then $a^{n}$ is a semi-primitive root mod $p$. 


$$
\begin{aligned}
& \Leftrightarrow\left(n, \frac{p-1}{2}\right)=1 \\
& \therefore S=\sum_{1 \leq n \leq \frac{p-1}{2}} a^{n} \bmod p
\end{aligned}
$$

But $\sum_{1 \leq n \leq \frac{p-1}{2}} a^{n}=\sum_{1 \leq n \leq \frac{p-1}{2}} a^{n} \quad \sum_{d / n \& d / \frac{p-1}{2}} \mu(d)=\sum_{d / \frac{p-1}{2}} \mu(d) a^{n \delta}$

$=\sum_{\delta \prec \frac{p-1}{2 d}}\left(a^{d}\right)^{\delta} \cdot \sum_{d / \frac{p-1}{2}} \mu(d)$

$=\mu\left(\frac{p-1}{2}\right) a^{\frac{p-1}{2}}+\sum_{\delta \prec \frac{p-1}{2 d}}\left(a^{d}\right)^{\delta} \sum_{d / \frac{p-1}{2}} \mu(d)$

$=\mu\left(\frac{p-1}{2}\right) a^{\frac{p-1}{2}}+\sum_{d / \frac{p-1}{2}} \mu(d) \frac{a^{d}\left(a^{\frac{p-1}{2}}-1\right)}{a^{d}-1}$

Since $d / \frac{p-1}{2}$ and $\frac{a^{\frac{p-1}{2}}-1}{a^{d}-1}$ is an integer we have $S=\mu\left(\frac{p-1}{2}\right)$ mod p.

If $\mathrm{p}$ is a prime of the form $8 \mathrm{n}+1$, then $\mu\left(\frac{p-1}{2}\right)=\mu(4 n)=0 . \therefore S=0$

We know that ' $\mathrm{a}$ ' is quadratic residue $\bmod \mathrm{p}$ if $\mathrm{x}^{2} \equiv \mathrm{a}(\bmod \mathrm{p})$ has a solution.

Theorem3: If ' $a$ ' is semi-primitive root mod $p$ then ' $a$ ' is a quadratic residue mod p. Proof: $\boldsymbol{A}$ is a semi primitive root $\bmod p \Rightarrow a^{\frac{p-1}{2}} \equiv 1 \bmod p$

Since $\mathrm{p}$ is an odd prime $\mathrm{p}$ has a primitive root say $\mathrm{g}$.

Now $(\mathrm{a}, \mathrm{p})=1$ we have $a \equiv g^{k} \bmod p ; 1 \leq k \leq \phi(p)$

$1 \equiv a^{\frac{p-1}{2}} \equiv\left(g^{k}\right)^{\frac{p-1}{2}}(\bmod p)$

$\Rightarrow \mathrm{p}-1$ divides $\mathrm{k} \cdot \frac{p-1}{2} \quad$ since $\mathrm{g}$ is a primitive root $\bmod \mathrm{p}$.

$\Rightarrow \frac{k}{2}$ is an integer i.e $\frac{k}{2}=m$

$\therefore a \equiv\left(g^{m}\right)^{2}(\bmod p)$

$\Rightarrow g^{m}$ is a solution of $\therefore x^{2} \equiv a(\bmod p)$ Therefore a is a quadratic residue mod $\mathrm{p}$.

However converse is not true as there are $\frac{\phi(\phi(p))}{2}$ semi-primitive roots and $\frac{p-1}{2}$ quadratic residues. 
Theorem4: If ' $a$ ' is a primitive root $\bmod p$ where $p=4 n+3$, then $-a$ is a semi primitive root $\bmod p$.

Proof: Let ' $a$ ' be a primitive root mod $\mathrm{p}$.

Then $a^{p-1} \equiv 1 \bmod p \Rightarrow(-a)^{p-1} \equiv 1 \bmod p$

$a^{p-1} \equiv 1 \bmod p \Rightarrow\left(a^{\frac{p-1}{2}}+1\right)\left(a^{\frac{p-1}{2}}-1\right) \equiv 0 \bmod p$

$\Rightarrow a^{\frac{p-1}{2}} \equiv-1 \bmod p$ as a is a primitive root $\bmod \mathrm{p}$.

$\Rightarrow(-a)^{\frac{p-1}{2}} \equiv 1 \bmod p$

Suppose $\exp _{\mathrm{m}}(-\mathrm{a})=\mathrm{f}$

Then $\mathrm{f} \mid \frac{p-1}{2}, 1 \leq \mathrm{f} \leq \frac{p-1}{2}$; i.e. $2 \mathrm{f}<\mathrm{p}-1$

Since $\exp _{\mathrm{m}}(-\mathrm{a})=\mathrm{f}$ we have $(-\mathrm{a})^{2 \mathrm{f}} \equiv 1(\bmod \mathrm{p})$

$\Rightarrow \mathrm{a}^{2 \mathrm{f}} \equiv 1(\bmod \mathrm{p})$ which is a contradiction since ' $\mathrm{a}$ ' is a primitive root mod $\mathrm{p}$.

Therefore $\mathrm{f}=\frac{p-1}{2}$

Hence - a is semi-primitive root mod $\mathrm{p}$.

Similarly we can prove that if ' $p$ ' is of the form $4 n+1$ then $\exp _{m}(-a)=p-1 / 4$ when $n$ is

odd.

Theorem5: If $8 \mathrm{n}-1$ and $4 \mathrm{n}-1$ are primes then 2 is a semi-primitive root mod $8 \mathrm{n}-1$.

Proof; Let $\mathrm{p}=8 \mathrm{n}-1$ and $\mathrm{q}=4 \mathrm{n}-1$.Then $\mathrm{p}-1=2 \mathrm{q}$.

From Gauss lemma we have

$\left(\frac{2}{p}\right) \equiv 2^{\frac{p-1}{2}}(\bmod p)$

And

$$
\begin{gathered}
\left(\frac{2}{p}\right)=(-1)^{\frac{p^{2}-1}{8}}=(-1)^{\frac{(8 n-1)^{2}-1}{8}}=(-1)^{8 n^{2}-2 n}=1 i \cdot 2^{\frac{p-1}{2}} \equiv 1(\bmod p) \\
\therefore 2^{\frac{p-1}{2}} \equiv 1(\bmod p)
\end{gathered}
$$

Suppose $\exp _{\mathrm{p}} 2=\mathrm{f}$ then $2^{\mathrm{f}} \equiv 1(\bmod \mathrm{p})$ and $\mathrm{f}$ divides $\mathrm{p}-1 / 2$ i.e $\mathrm{f} \mid \mathrm{q}$

Since $\mathrm{q}$ is a prime we have $\mathrm{f}=\mathrm{q}$.

Thus 2 is a semi-primitive root mod $\mathrm{p}$.

Theorem 6: ' 2 ' is a semi primitive root $\bmod \mathrm{p}$, where $\mathrm{p}=2 \mathrm{q}+1$, $\mathrm{q}$ being an odd prime of the form $4 n+3$. 
Proof: Let $\mathrm{p}=2 \mathrm{q}+1$ then $\mathrm{p}-1 / 2=\mathrm{q}$ where $\mathrm{q}=4 \mathrm{n}+3$

$$
\begin{gathered}
\left(\frac{2}{p}\right)=(-1)^{\frac{p^{2}-1}{8}}=(-1)^{\frac{(2 q+1)^{2}-1}{8}}=(-1)^{\frac{4 q^{2}+4 q}{8}}=(-1)^{\frac{4(4 n+3)^{2}+4(4 n+3)}{8}}=(-1)^{8 n^{2}+14 n+6}=1 \\
\therefore 2^{\frac{p-1}{2}} \equiv 1(\bmod p)
\end{gathered}
$$

By Gauss lemma,

Suppose $\exp _{\mathrm{p}} 2=\mathrm{f}$ then $2^{\mathrm{f}} \equiv 1(\bmod \mathrm{p})$ and $f$ divides $\mathrm{p}-1 / 2$ i.e. $f \mid \mathrm{q}$

Since $\mathrm{q}$ is a prime we have $f=\mathrm{q}$.

Thus 2 is a semi-primitive root mod $\mathrm{p}$.

Theorem7: If $8 n+3$ and $4 n+1$ are primes then -2 is a semi-primitive root mod $8 n+3$.

Proof: let $p=8 n+3$ and $q=4 n+1$ So $p-1 / 2=4 n+1$.

By Gauss lemma

$$
\begin{aligned}
& \left(\frac{2}{p}\right)=(-1)^{\frac{p^{2}-1}{8}}=(-1)^{\frac{(8 n+3)^{2}-1}{8}}=(-1)^{8 n^{2}+6 n+1}=(-1)^{(4 n+1)(2 n+1)}=-1 \\
& \therefore 2^{\frac{p-1}{2}} \equiv-1(\bmod p) \text { hence }(-2)^{\frac{p-1}{2}} \equiv 1 \bmod p
\end{aligned}
$$

Suppose $\exp _{\mathrm{p}}(-2)=\mathrm{f}$ then $(-2)^{\mathrm{f}} \equiv 1(\bmod \mathrm{p})$ and $f$ divides $\mathrm{p}-1 / 2$ i.e $f \mid \mathrm{q}$

Since $\mathrm{q}$ is a prime we have $f=\mathrm{q}$

Thus -2 is a semi-primitive root mod $\mathrm{p}$.

Key Words: Universal exponent, exponent, $\lambda$ - primitive root, semi-primitive root, primitive root.

\section{References:}

[1] Apostol Tom M: Introduction to Analytic Number Theory, Springer International Student Edition.

[2] Leveque W.J.: Topics in Number Theory, Vol. 1 Reading mass (1956).

[3] Vegh E: $\lambda$ - primitive roots. Portugaliae Mathematica 40(1981) 297-303.

[4] An Introduction to Theory of Numbers by Ivan Niven, Herbert S. Zuckerman - Wiley Eastern Limited.

[5] Elementary Number theory by David M .Burton. 